\title{
Abnormal Brain Network in Epilepsy and Associated Comorbidites
}

\author{
Huajun Yang ${ }^{1,2,3}$, Jiechuan Ren ${ }^{1,2,3}$, Qun Wang ${ }^{1,2,3,+}$
}

\begin{abstract}
Epilepsy has been recognized as a brain network disorder. With the development of techniques investigating structural and functional brain network, current studies provided insights into the physiopathogenesis of the epilepsy, including focal epilepsy and idiopathic generalized epilepsy. Besides, a number of studies have shed light on the effects of epilepsy on other functional networks, which help to explain cognitive impairment and psychiatric disorders comorbid with epilepsy. In this review, we will discuss current studies that provide structural and functional network evidence of epilepsy or its cognitive and psychiatric comorbidities. Clinical features and outcome of epilepsy result from changes in the networks, which are associated with the clinical course of the disease, the presence of complications and the location of the epileptogenic zone. Different network changes and remodeling are closely related to the efficacy of treatment and prognosis of epilepsy. However, knowledge regarding network mechanism in epilepsy remains limited at present. Further validation through novel investigating techniques data analysis methods are required for its reliable application in the clinical management of individual patients.
\end{abstract}

\section{Key words}

Epilepsy, Comorbidity, Brain network, Cognition, Psychiatry

\section{Abbreviations}

EEG: Electroencephalogram; TLE: Temporal Lobe Epilepsy; Mtle: Mesial Temporal Lobe Epilepsy; DTI: Diffusion Tensor Imaging; MD: Mean Diffusivity; FA: Fractional Anisotropy; HS: Hippocampal Sclerosis; FC: Functional Connectivity; MRI: Magnetic Resonance Imaging; Fmri: Functional Magnetic Resonance Imaging; BOLD: Blood Oxygen Level Dependent; SPECT: Single PhotonemissionComputed Tomography; SISCOM: Subtraction Ictal-SPECT Coregistered To MRI; FLE: Frontal Lobe Epilepsy; SMA: Supplementary Motor Area; IGE: Idiopathic Generalized Epilepsy; AE: Absence Epilepsy; ME: Myoclonic
Epilepsy; GTCS: Generalized Tonic-Clonic Seizure; GSWD: Generalized Spike-And -Wave Discharge; VBM: Voxel Based Morphology; GMV: Gray Matter Volume; VL: Ventral Lateral Nucleus; MEG: Magnetoencephalography; DMN: Default Mode Network; ATN: Attention Network; FPN: Frontoparietal Control Network; Icns: Intrinsic Connectivity Networks; IGL: Intergeniculate Leaflet; CAE: Childhood Absence Epilepsy; JME: Juvenile Myoclonic Epilepsy; ACC: Anterior Cingulate Cortex; SWD: Spike-And-Wave Discharge; VPA: Valproic Acid; SPGR: Spoiled Gradient Recall 


\begin{abstract}
Introduction
Epilepsy is a common chronic disease of the nervous system, which produces a series of characteristic clinical symptoms and comorbidities due to brain structural and functional disturbances resulting from prolonged, repetitive, and abnormal firing of brain neurons. In recent years, epilepsy has been conceptualized recently as a disorder involving networks, rather than single sources of pathology in the human brain [1]. Spencer [2] has defined epileptic network as: bilateral cerebral cortex and subcortical structures that are connected anatomically and functionally; any part of the activity will affect other structures and, as a whole, produce clinical manifestations and EEG features associated with epileptic seizures. The main methods used in epilepsy network research are as follows: (1) Structural networks, including the composition of neurons and physical connections between the brain regions; (2) Functional networks, representing a dynamic combination of independent regions of the brain, including metabolic functions, i.e., normal and abnormal metabolism in related regions [3]. By understanding the network mechanisms of epilepsy and its comorbidities, we can identify the main origin of epilepsy in the network and predict the clinical course and long-term outcome, which helps selecting appropriate therapies to prevent the effect of abnormal network. We will review current studies that provide structural and functional network evidence of epilepsy or its cognitive and psychiatric comorbidities.
\end{abstract}

\section{Abnormal Networks and Pathophysiology of Epilepsy}

\section{- Focal epilepsies}

Researchers have increasingly suggested that focal epilepsies are associated to abnormal brain function within an epileptic network, rather than dysfunction in a single epileptogenic region.

\section{- Temporal lobe epilepsy}

The majority of studies examining structural and functional network in focal epilepsies have focused on temporal lobe epilepsy (TLE), which is the most common focal epilepsy in adults.

Conventional MRI has been used to study the pathological changes of temporal lobe epilepsy, such as gray matter atrophy, white matter reduction and cortical thickness change. In addition to hippocampal atrophy and sclerosis, which is the most common pathological change in mesial temporal lobe epilepsy (mTLE), a large number of studies have revealed changes in the cortex in temporal and extra-temporal regions, including volume, morphology, cortical thickness and structure $[4,5]$. Researchers have found reduction of white matter associated with ipsilateral temporal lobe in epileptic foci and the thickness of olfactory and orbital cortex was positively correlated with the thickness of temporal and frontal cortex [6,7]. Diffusion tensor imaging (DTI) reflects the fiber density of fiber and network nodes in different brain regions. Bonilha et al. [8] found a decrease in fiber density in the related area of patients with mesial temporal lobe epilepsy. On the contrary, the fiber density increases in the limbic network. Sharon et al. [9] found that changes in hippocampus and cingulate gyrus were more prominent in right temporal lobe epilepsy while changes in remote area were more prominent in left temporal lobe epilepsy. Increase in the mean diffusivity (MD) of right hippocampus and decrease in the fractional anisotropy of the left lateral capsule could predict lateralization of TLE. This change is related to the duration of epilepsy, and the white matter pathway is easily influenced by seizure recurrence. Similar findings were observed in animal model of mTLE [10]. The epileptogenic rats exhibited significant changes in DTI-measured fractional anisotropy (FA) in numerous brain regions versus nonepileptogenic rats. Changes included decreases and increases in FA in regions such as the entorhinal-hippocampal area, amygdala, corpus callosum, thalamus, striatum, accumbens, and neocortex. The FA changes evolved over time as animals transitioned from early to late epileptogenesis. In mTLE patients, the network structure of hippocampal sclerosis (HS) lesions is different from that in patients without definite pathological foci. Trotta et al. [11] found that in patients with unilateral temporal lobe epilepsy, connectivity between amygdala, ventral lateral prefrontal cortex, temporal pole, and cingulate cortex in non-epileptic side significantly decreased while connectivity in midline structures such as ventral lateral prefrontal and anterior cingulate cortex, dorsal medial prefrontal cortex and the temporo parietal junction connections increased. Abnormal uncinate and arcuate fasciculus tracts were also observed in mTLE patients, which are related to language pathway [12].

Functional connectivity researchers have confirmed the involvement of widespread 
brain networks in TLE [13]. In several studies, decreased functional connectivity was observed in the ipsilateral hemisphere while increases were found in contralateral mesiotemporal networks $[14,15]$. However, in some other studies, functional connectivity analysis showed that functional connectivity between epileptic focus and ipsilateral hemisphere increased during interictal period ,involving hypothalamus, temporal lobe, insula, frontal lobe, basal ganglia, angular gyrus, cerebellum and brainstem $[16,17]$. Kucukboyaci et al. [18] reported extensive regional FC reductions during the interictal period, including reductions in the ipsilateral hippocampus and parahippocampal gyrus, which can be found in both sides, but more prominent in the epileptic side. These mixed findings of altered functional connectivity within temporo-limbic circuits in TLE may help to explain varied seizure semiology. Liao et al. [19] found increased connections within the mesial temporal lobe but decreased connectivity to extratemporal areas in TLE. Tracy et al. [20] reported that in unilateral temporal lobe epilepsy, decrease in activity in extratemporal areas could be observed in either side, which was more significant in the contralateral hemisphere. TLE patients lack strong inhibition of brain activity in the contralateral hemisphere when extratemporal interictal activities were displayed. Such inhibition may be adaptive by constraining epileptiform activity to the epileptogenic temporal lobe. This finding might explain the poor prognosis of TLE patients with bilateral interictal activity, as they lack such beneficial inhibition in functional network. Functional MRI (fMRI) can be used to explore the relationship between brain activation and functioning. In fMRI, changes in the blood oxygen level dependent (BOLD) signal accompany neural activity in the brain. Functional connectivity studies based on EEGfMRI were conducted to determine the influence of interictal epileptic activity on determined functional networks. Researchers have proposed an independent component analysis of functional connectivity based on EEG-fMRI and a variety of classifiers that exclusively detects a single map related to interictal epileptic brain activity [21]. This method yields functional connectivity maps that correctly identify the epileptogenic zone in several EEG-negative cases while simultaneously maintaining high specificity. Recent study [22] also reported that the overlap between hyperperfusion on subtraction ictal-SPECT coregistered to MRI (SISCOM) and positive BOLD responses while between hypoperfusion and negative BOLD responses within the same network on EEG-fMRI, which suggested good concordance between the irritative zone and the seizure onset zone. It might be a promising method to localize and track the evolution of epileptogenic activity.

\section{- Frontal lobe epilepsy}

Frontal lobe epilepsy (FLE) is the second most common form of focal epilepsy [1]. Patients with FLE exhibit motor symptoms during the ictal period and deficits in motor control, planning, and coordination during the interictal period [23]. Widjaja et al. [24] conducted a study on structural networks in children with localization-related epilepsy. In the study they found disruptions in global and regional networks and subnetworks in children with FLE, and the frontal lobe epilepsy subgroup demonstrated more areas with reduced nodal efficiency and more impaired subnetworks than in TLE. However, a study on the connectivity of the supplementary motor area in FLE using DTI and probabilistic tractography reported that in FLE, the structural connectivity of the supplementary motor area (SMA) was preserved, suggesting a robust motor network that is not compromised by longstanding epilepsy involving the medial frontal lobes [25]. This has important implications for epilepsy surgery planning in the pericentral cortex and is also consistent with the lack of functional motor deficit in most patients with FLE without a motor cortex lesion. As for the functional networks, researchers have reported decreased connectivity within the motor network in patients with FLE, which was in association with number of lifetime seizures [26]. Besides, Woodward et al. [27] reported that patients with FLE who experienced a recent seizure relied more on the sensorimotor cortex of the contralateral hemisphere during fingertapping and coordination tasks than those with lower seizure frequency, as evidenced by fMRI. In spite of limited sample sizes and divergent data, these studies have provided descriptions of functional cortical motor organization in patients with FLE [28].

\section{- Idiopathic generalized epilepsies}

Idiopathic generalized epilepsies (IGE), including absence epilepsy (AE), myoclonic epilepsy (ME), and generalized tonic-clonic seizures (GTCS), account for approximately $20 \%$ of epilepsy diagnoses [1]. The current view on epilepsy classification demonstrates that generalized epilepsy originates from a point, and 
quickly spread through the cortex and subcortical network transmission in bilateral hemispheres [29]. Thalamocortical dysfunction is considered to be the major mechanism of IGE [1]. Abnormal interactions between cortical and thalamic cause generalized spike-and -wave discharge (GSWD), which has been clearly established by in vitro and in vivo animal studies [30], although the definite origin of IGE and roles of cortices and thalamus are still controversial. However, a more definitive answer regarding the generalized epileptic neuronal firing patterns needs further verification in patients.

The developments of neuroimaging and electromagnetic physiology methods have promoted studies on the neural network of IGE. Conventional MRI usually cannot show structural abnormalities of brain network in patients with IGE. Voxel based morphology (VBM) can detect subtle alterations in brain structure without prior assumptions. VBM studies [31] on IGE demonstrated significant gray matter volume (GMV) increase in right ventral lateral nucleus (VL) and right medial frontal gyrus, and significant GMV decrease in bilateral pulvinar, which contributed to the disordered neural activities in thalamocortical circuits. Bernhardt et al. [32] found that the thickness of the cortex is positively related to the volume of thalamus, and that the thickness of the frontal cortex in patients with IGE is significantly decreased, suggesting that generalized seizures cause remodeling of the thalamocortical structure. DTl studies [33-35] revealed structural abnormalities in the thalamus and basal ganglia in patients with IGE, and these abnormalities correlated with the course of seizures, which provided structural evidence for thalamocortical network abnormalities. In recent years, researchers have paid more and more attention to the research of functional networks. Rajaei et al. [36] used EEG functional connectivity maps to investigate and compare different types of epileptiform activity. Results showed some differences between connectivity patterns of single spikes related to focal epilepsy and connectivity patterns of repetitive spikes related to generalized epilepsy, which support the theory that higher global connections are seen in generalized epilepsy rather than the more local connections observed in the case of focal epilepsy. Magnetoencephalography (MEG) study [37] revealed a widespread increase in functional network connectivity in the resting state in IGE, with a particular focus on the motor network but clearly extending beyond this, which could be related to an increased likelihood to generate spontaneous, generalized seizures in patients. The fMRI functional connectivity method provided a map of the neural networks needed for seizure generation, and demonstrated ictal and interictal disturbances in normal physiological networks [38]. A study of baboon model of Idiopathic generalized epilepsy [39] demonstrated significant increases in the occipital gyri of the epileptic group's intrinsic functional connectivity map for the default mode network (DMN), cingulate, intraparietal, motor, visual, amygdala, and thalamic regions. Wei et al. [40] explored the functional connectivity between the subnetworks of the DMN, attention network (ATN), and frontoparietal control network (FPN) using independent component analysis of resting-state fMRI data collected from 27 patients with IGE. Their findings suggested that IGE was likely associated with a disrupted brain organization probably derived from abnormal functional interactions among intrinsic connectivity networks (ICNs). Furthermore, the changes in the functional architecture of the ICNs might be associated with deficits in attention and mentation in IGE, which provided evidence for understanding pathophysiology of IGE [40].

Absence epilepsy (AE) is a disease characterised by spike-wave discharges present in the encephalogram, directly caused by hypersynchronous thalamo-cortical oscillations. in vitro patch-clamp study on WAG/Rij rat model of absence epilepsy [41] showed higher firing rate of infra-slow oscillatory intergeniculate leaflet (IGL) neurons with an abnormal reaction to a change in constant illumination, which suIGEsted disinhibition of the IGL network. Additional immunohistochemical experiments indicated astrogliosis in the area of the IGL, which might partially underlie the observed changes in inhibition. In patients of AE, VBM study showed that the most significant GMV increase was found in right VL, and slight GMV reduction was seen in right medial dorsal nucleus, right subcallosal gyrus, left caudate and left precuneus [31]. DTI study [42] indicated that structural impairments existed in DMN regions in children suffering from AE. EEGfMRI has become one of the dominant means of studying the functional pathophysiology of GSWD in absence epilepsy. Blumenfeld et al. [43] proposed a network inhibition hypothesis in which they correlated the inhibition of sub- 
cortical activating systems with awareness loss during seizures through disruptions in their interactions with the DMN. Another study [44] showed early change in the BOLD signal in the DMN, which demonstrated that either activity in the DMN initiated the GSWD in AS or the DMN played a certain role in permitting or facilitating the occurrence of epileptiform events. In addition to the DMN, the thalamus also has an important role in AS generation. EEG-fMRI studies [44,45] revealed a positive thalamic BOLD response which was consistently associated with the GSWD and absence seizures. Alterations of resting-state network in the frontal lobe cortex were also reported in studies on $\mathrm{AE}$ [46]. Besides, a multi-frequency MEG study [47] showed that CAE patients displayed frequencyspecific abnormalities in the network pattern even during the inter-ictal period, and the frontal cortex and precuneus/posterior cingulate cortex might play crucial roles in the pathophysiology of CAE. The effective connectivity network of CAE patients was overconnective and random during the inter-ictal period.

Juvenile myoclonic epilepsy (JME) is characterized by myoclonic jerks, generalized tonic-clonic seizures, and, less frequently, absence seizures. For JME, significant GMV increase was seen in right medial frontal gyrus, right anterior cingulate cortex (ACC), while significant GMV decrease was found in right pulvinar in VBM study [31], which provided evidence for thalamocortical abnormality. DTI study [25] showed that structural connectivity of the SMA was significantly reduced in JME compared to controls. Functional network abnormalities and underlying mechanisms of JME were investigated with the development of functional connectivity techniques. A study of animal model of myoclonic seizures [48] showed that EEG connectivity among all electrode pairs increased at the onset of seizures. In an EEG study on spike-and-wave discharge (SWD) in JME [49] used graph theoretical measures, including nodal degree, characteristic path length, clustering coefficient, and smallworld index to evaluate the characteristics of epileptic networks in JME. The nodal degree of the SWD network, particularly in the frontal region, was significantly higher compared to the baseline network. Long-range connections were increased during SWD, particularly between frontal and posterior brain regions. In a fMRI study involving a working memory paradigm [50], the researchers found increased functional connectivity between the motor system and areas of higher cognitive functions within the frontal and the parietal lobes, which might explained clinical feature that myoclonic jerks could be triggered by cognitive effort in some patients with JME [51]. Additionally, in patients under valproic acid (VPA) treatment, abnormal motor cortex co-activation was negatively associated with an increasing daily VPA dose, which might underlie the beneficial effect of VPA on controlling myoclonic jerks [50].

\section{- Abnormal networks and neurocognitive and psychiatric comorbidities}

In addition to exploring underlying mechanisms of epilepsy, connectivity analysis can also be utilized to investigate the interaction between the epileptic network and neurocognitive networks underlying neuropsychiatric comorbidities in patients with epilepsy. Patients with chronic epilepsy frequently display cognitive comorbidity and might have widespread network abnormalities outside the epileptic zone, which might affect a variety of cognitive functions and global intelligence [52]. In previous studies on structural network abnormalities in epilepsy with cognitive impairment, Volumetric MRI and VBM have revealed significant volume losses in the area of the seizure focus as well as in distant areas [53,54]. DTI adds evidence of loss of integrity of connections from the seizure focus to distant areas as well as between distant areas $[10,55]$. A study [52] collected DTI data from 39 patients with nonsymptomatic localization-related epilepsy and varying degrees of cognitive impairment and 23 age-matched healthy controls. Weighted graph theoretical analysis showed Patients with severe cognitive impairment showed lower clustering (a measure of brain network segregation) and higher path length (a measure of brain network integration) compared with the healthy controls and patients with little or no cognitive impairment. $\mathrm{AMRI}$ is considered as a main method to investigate functional network abnormalities in neurocognitive comorbidities of epilepsy. The majority of fMRI researchers have focused on the influence of TLE on resting-state networks, which has important implications for understanding higher cognitive function impairments. TLE is one of the major risk factors for memory decline [56]. Voets et al. [57] reported a decline in functional connectivity between bilateral mesial temporal lobes, occipital lobes and left orbitofrontal regions involved in memory processing in patients with left TLE. Another resting fMRI study [58] suggested 
aberrant connectivity within anterior and posterior hippocampal-cortical networks and altered signalling during task performance, which was related to memory decline. Li et al. [59] found that left and right mTLE exhibited different neural reorganization patterns of anterior and posterior hippocampal networks associated with verbal memory. Fan et al. [60] suggested that weakened contralateral connection and disrupted effective interaction between subregions of the unitary, transmodal hub of the ATL may be the primary cause of anomia in the left HS patients. Moreover, researchers found that the functional connectivity between nonpathological medial temporal lobe and medial frontal cortex was related to memory function preservation, which might be a compensatory mechanism in patients with TLE $[15,61]$. A combined language task and resting-state fMRI study [62] showed decreased connectivity in left TLE, which explained language disturbance in patients. Language-paradigm fMRI studies $[63,64]$ revealed a right shift of language reorganization network in patients with left TLE. Besides TLE, less interconnected subnetworks were found in cognitively impaired children with FLE [65]. In patients with JME, reduced functional connectivity within the prefrontal cortex was associated with frontal dysfunction characterized by impulsivity, impatience and social immaturity $[23,66]$. In rolandic epilepsy, transient influences of interictal centrotemporal spikes on functional networks were responsible for cognition and language dysfunction, which was evidenced by EEG-fMRI [67].

Psychiatric comorbidities in epilepsy are of great concern, which influences quality of life in patients with epilepsy. As many as 20\%-55\% of TLE patients suffer from depression [68]. A DTI study [69] showed that anatomical connections, which were diminished both in the left and right mTLE, were primarily located in the limbicfrontal network, partially agreeing with the limbic-frontal dysregulation model of depression. This may suggest the neuropathological mechanisms underlying the comorbidity of depression in mTLE. Alterations in the frontolimbic network in TLE patients with depressive symptoms were also observed in functional connectivity studies [70]. In another study [71], the researchers demonstrated that hippocampalanterior prefrontal functional connectivity contributed strongly to depressive symptoms in the left TLE group compared to the right TLE group. Right amygdala functional connectivity was associated with depressive symptoms in both left and right TLE. These findings suggested that the right hemisphere might have a greater influence on emotional networks and play a greater role in psychiatric commobidites. In addition, a graph theory analysis based on T1weighted 3D spoiled gradient recall (SPGR) showed large scale disruptions in cortical and subcortical brain regions in children with recent onset epilepsy and anxiety [72]. Influence in the network is not necessarily local but potentially influencing other more distant regions [71]. All these findings may help provide new insight into mood disorders in epilepsy that could eventually guide treatment planning.

\section{n Perspective}

Current studies on structural and functional networks have provided invaluable insights into the multifocal aspect of the neuronal network underlying epilepsy and its comorbidities. Some clinical features and interictal complications of epilepsy result from changes in the networks, which are associated with the clinical course of the disease, the presence of complications and the location of the epileptogenic zone. Different network changes and remodeling are closely related to the efficacy of treatment and prognosis of epilepsy. However, the current evidence is still far from enough to be applied to clinical practice due to limited sample size and divergent data. Therefore further studies remains necessary with the development of a variety of noninvasive and reproducible investigating techniques.

A combination of several techniques by multimodal measurements may be most informative method to explore neuronal correlates of epilepsy and its comorbidities. For example, by combining fMRI and DTI techniques, the functional impact of white matter tract abnormalities or the impact of functional impairments on white matter tract integrity can be evaluated. Then the hypotheses about altered structure-function relationships accounting for epilepsy can be validated. Additionally, new methods of data analysis may be helpful in combined analyses of different techniques. For example, graph theoretical analysis is a technique that converts fMRI, EEG, MEG data to abstract graphs to investigate the level of interaction between distinct brain regions [73].

In the future, the development in these novel techniques will help to explore the pathogenesis of epilepsy, accurately locate epilepsy foci and record dissemination of epileptic activities, in 
order to predict the clinical course and outcome of epilepsy establish individualized treatment strategies and improve the quality of life in patients with epilepsy.

\section{Declaring of Conflicting Interests}

None of the authors have any conflicts of interests to disclose.

Acknowledgements
This study was supported by the National Natural
Science Foundation (NSFC 8160050105) and
the Capital Health Research and Development of
Special (2016-1-2011).

\section{References}

1. Berg AT, Berkovic SF, Brodie MJ, et al. Revised terminology and concepts for organization of seizures and epilepsies: report of the ILAE Commission on Classification and Terminology, 2005-2009. Epilepsia 51(4), 676-685 (2010).

2. Spencer SS. Neural networks in human epilepsy, evidence of and implications for treatment. Epilepsia 43(3), 219-227 (2002).

3. Kramer MA, Cash SS. Epilepsy as a disorder of cortical network organization. Neuroscientist 18(4), 360-372 (2012).

4. Bernhardt $\mathrm{BC}$, Bernasconi $\mathrm{N}$, Concha $\mathrm{L}$, et al Cortical thickness analysis in temporal lobe epilepsy: reproducibility and relation to outcome. Neurology 74(22), 1776-1784 (2010).

5. Bernhardt BC, Bernasconi N, Kim H, et al. Mapping thalamocortical network pathology in temporal lobe epilepsy. Neurology 78(2), 129-136 (2012)

6. Bernasconi N, Duchesne S, Janke A, et al. Whole-brain voxel-based statistical analysis of gray matter and white matter in temporal lobe epilepsy. Neuroimage 23(2), 717-723 (2004).

7. Bernhardt BC, Worsley $\mathrm{KJ}$, Besson $\mathrm{P}$, et al. Mapping limbic network organization in temporal lobe epilepsy using morphometric correlations: insights on the relation between mesiotemporal connectivity and cortical atrophy. Neuroimage 42(2), 515-524 (2008).

8. Bonilha L, Nesland T, Martz GU, et al. Medial temporal lobe epilepsy is associated with neuronal fibre loss and paradoxical increase in structural connectivity of limbic structures. J. Neurol. Neurosurg. Psychiatry 83(9), 903-909 (2012).

9. Chiang $\mathrm{S}$, Levin HS, Wilde $\mathrm{E}$, et al. White matter structural connectivity changes correlate with epilepsy duration in temporal lobe epilepsy. Epilepsy. Res 120(1), 37-46 (2016).

10. Wang $\mathrm{H}$, Huang $\mathrm{Y}$, Coman $\mathrm{D}$, et al. Network evolution in mesial temporal lobe epilepsy revealed by diffusion tensor imaging. Epilepsia 58(5), 824-834 (2017).

11. Trotta N, Goldman S, Legros B, et al. Changes in functional integration with the non-epileptic temporal lobe of patients with unilateral mesiotemporal epilepsy. PLoS. One 8(6), e67053 (2013).
12. Bonilha L, Martz GU, Glazier SS, et al. Subtypes of medial temporal lobe epilepsy: influence on temporal lobectomy outcomes? Epilepsia 53(1), 1-6 (2012).

13. Cataldi M, Avoli M, de Villers-Sidani E. Resting state networks in temporal lobe epilepsy. Epilepsia 54(12), 2048-2059 (2013).

14. Bettus G, Bartolomei F, Confort-Gouny S, et al. Role of resting state functional connectivity MRI in presurgical investigation of mesial temporal lobe epilepsy. J. Neurol. Neurosurg. Psychiatry 81(10), 1147-1154 (2010).

15. Bettus G, Guedj E, Joyeux F, et al. Decreased basal fMRI functional connectivity in epileptogenic networks and contralateral compensatory mechanisms. Hum. Brain. Mapp 30(5), 1580-1591 (2009).

16. Haneef Z, Chiang S, Yeh HJ, et al. Functional connectivity homogeneity correlates with duration of temporal lobe epilepsy. Epilepsy. Behav 46(1), 227-233 (2015).

17. Haneef $\mathrm{Z}$, Lenartowicz A, Yeh HJ, et al. Functional connectivity of hippocampal networks in temporal lobe epilepsy. Epilepsia 55(1), 137-145 (2014).

18. Kucukboyaci NE, Kemmotsu N, Cheng CE, et al. Functional connectivity of the hippocampus in temporal lobe epilepsy, feasibility of a task-regressed seed-based approach. Brain. Connect 3(5), 464-474 (2013).

19. Liao W, Zhang Z, Pan Z, et al. Altered func tional connectivity and small-world in mesial temporal lobe epilepsy. PLoS. One 5(1), e8525 (2010).

20. Tracy Jl, Osipowicz K, Spechler P, et al. Functional connectivity evidence of cortico-cortico inhibition in temporal lobe epilepsy. Hum. Brain. Mapp 35(1), 353-366 (2014).

21. Hunyadi $B$, Tousseyn $S$, Dupont $P$, et al. A prospective fMRI-based technique for localising the epileptogenic zone in presurgical evaluation of epilepsy. Neuroimage 113(1), 329-339 (2015).

22. Tousseyn S, Dupont P, Goffin K, et al. Correspondence between large-scale ictal and interictal epileptic networks revealed by single photon emission computed tomography (SPECT) and electroencephalography (EEG)-functional magnetic resonance imaging (fMRI). Epilepsia 56(3), 382-392 (2015).

23. Wandschneider B, Thompson PJ, Vollmar C, et al. Frontal lobe function and structure in juvenile myoclonic epilepsy, a comprehensive review of neuropsychological and imaging data. Epilepsia 53(12), 2091-2098 (2012).

24. Widjaja E, Zamyadi M, Raybaud C, et al. Disrupted Global and Regional Structural Networks and Subnetworks in Children with Localization-Related Epilepsy. AJNR. Am. J. Neuroradiol 36(7), 1362-1368 (2015).

25. Vulliemoz S, Vollmar C, Koepp MJ, et al. Connectivity of the supplementary motor area in juvenile myoclonic epilepsy and frontal lobe epilepsy. Epilepsia 52(3), 507-514 (2011).

26. Woodward KE, Gaxiola-Valdez I, Goodyear BG, et al. Frontal lobe epilepsy alters functional connections within the brain's motor network, a resting-state fMRI study. Brain. Connect 4(2), 91-99 (2014).

27. Woodward KE, Gaxiola-Valdez I, Mainprize D, et al. Recent seizure activity alters motor organization in frontal lobe epilepsy as revealed by task-based fMRI. Epilepsy. Res 108(8), 12861298 (2014).

28. Xiao F, An D, Zhou D. Functional MRI-based connectivity analysis, A promising tool for the investigation of the pathophysiology and comorbidity of epilepsy. Seizure 44(1), 37-41 (2017).

29. Meeren H, van Luijtelaar G, Lopes da Silva F, et al. Evolving concepts on the pathophysiology of absence seizures, the cortical focus theory. Arch. Neurol 62(3), 371-376 (2005).

30. Huguenard JR, McCormick DA. Thalamic synchrony and dynamic regulation of global forebrain oscillations. Trends. Neurosci 30(7), 350-356 (2007).

31. G Bin, T Wang, $\mathrm{H}$ Zeng, et al. Patterns of Gray Matter Abnormalities in Idiopathic Generalized Epilepsy, A Meta-Analysis of Voxel-Based Morphology Studies. PLoS. One 12(1), e0169076 (2017).

32. Bernhardt BC, Rozen DA, Worsley KJ, et al. Thalamo-cortical network pathology in idiopathic generalized epilepsy, insights from MRI-based morphometric correlation analysis. Neuroimage 46(2), 373-381 (2009).

33. Liu M, Concha L, Beaulieu C, et al. Distinct white matter abnormalities in different idiopathic generalized epilepsy syndromes. Epilepsia 52(12), 2267-275 (2011). 
etry abnormalities in subcortical nuclei of patients with absence seizures. Epilepsia 52(6), 1092-1099 (2011).

35. Zhang Z, Liao W, Chen $\mathrm{H}$, et al. Altered functional-structural coupling of large-scale brain networks in idiopathic generalized epilepsy. Brain 134(Pt 10), 2912-2928 (2011).

36. Rajaei $H$, Cabrerizo $M$, Janwattanapong $P$, et al. Connectivity maps of different types of epileptogenic patterns. Conf Proc IEEE. Eng. Med. Biol. Soc 2016(1), 1018-1021 (2016).

37. Elshahabi A, Klamer S, Sahib AK, et al. Magnetoencephalography Reveals a Widespread Increase in Network Connectivity in Idiopathic/Genetic Generalized Epilepsy. PLoS. One 10(9), e0138119 (2015)

38. Gotman J, Grova C, Bagshaw A, et al. Generalized epileptic discharges show thalamocortical activation and suspension of the default state of the brain. Proc. Natl. Acad. Sci.U S A 102(42), 15236-15240 (2005).

39. Salinas FS, Szabo CA. Resting-state functional connectivity in the baboon model of genetic generalized epilepsy. Epilepsia 56(10), 1580-1589 (2015).

40. Wei HL, An J, Zeng LL, et al. Altered functional connectivity among default, attention, and control networks in idiopathic generalized epilepsy. Epilepsy. Behav 46(1), 118-125 (2015)

41. Chrobok L, Palus K, Jeczmien-Lazur JS, et al. Disinhibition of the intergeniculate leaflet network in the WAG/Rij rat model of absence epilepsy. Exp. Neurol 289(1), 103116 (2017).

42. Qiu W, Gao Y, Yu C, et al. Structural Abnormalities in Childhood Absence Epilepsy, Voxel-Based Analysis Using Diffusion Tensor Imaging. Front. Hum. Neurosci 10(1), 483 (2016).

43. Blumenfeld $\mathrm{H}$. Impaired consciousness in epilepsy. Lancet Neurol 11(9), 814-826 (2012).

44. Li Q, Luo C, Yang T, et al. EEG-fMRI study on the interictal and ictal generalized spikewave discharges in patients with childhood absence epilepsy. Epilepsy. Res 87(2-3), 160-168 (2009).

45. Carney PW, Masterton RA, Harvey AS, et al. The core network in absence epilepsy. Differences in cortical and thalamic BOLD response. Neurology 75(10), 904-911 (2010).

46. Killory BD, Bai X, Negishi M, et al. Impaired attention and network connectivity in childhood absence epilepsy. Neuroimage 56(4), 2209-2217 (2011).

47. Wu C, Xiang J, Jiang W, et al. Altered Effective Connectivity Network in Childhood
Absence Epilepsy, A Multi-frequency MEG Study. Brain. Topogr (2017).

48. Ding L, Gallagher MJ. Dynamics of sensorimotor cortex activation during absence and myoclonic seizures in a mouse model of juvenile myoclonic epilepsy. Epilepsia 57(10), 1568-1580 (2016).

49. Lee C, Im CH, Koo YS, et al. Altered Network Characteristics of Spike-Wave Discharges in Juvenile Myoclonic Epilepsy. Clin. EEG. Neurosci 48(2), 111-117 (2017).

50. Vollmar C, O'Muircheartaigh J, Barker GJ, et al. Motor system hyperconnectivity in juvenile myoclonic epilepsy, a cognitive functional magnetic resonance imaging study. Brain 134(Pt 6), 1710-1719 (2011)

51. Ferlazzo E, Zifkin BG, Andermann E, et al. Cortical triggers in generalized reflex seizures and epilepsies. Brain 128(Pt 4), 700-710 (2005).

52. Vaessen MJ, Jansen JF, Vlooswijk MC, et al. White matter network abnormalities are associated with cognitive decline in chronic epilepsy. Cereb. Cortex 22(9), 2139-2147 (2012).

53. Seidenberg M, Hermann B, Pulsipher D, et al. Thalamic atrophy and cognition in unilateral temporal lobe epilepsy. J. Int. Neuropsychol. Soc 14(3), 384-393 (2008).

54. Tosun D, Caplan R, Siddarth P, et al. Intelligence and cortical thickness in children with complex partial seizures. Neuroimage 57(2), 337-345 (2011).

55. Kucukboyaci NE, Girard HM, Hagler DJ, et al. Role of frontotemporal fiber tract integrity in task-switching performance of healthy controls and patients with temporal lobe epilepsy. J. Int. Neuropsychol. Soc 18(1), 57-67 (2012).

56. Thompson PJ, Conn H, Baxendale SA, et al. Optimizing memory function in temporal lobe epilepsy. Seizure 38(1), 68-74 (2016).

57. Voets NL, Adcock JE, Stacey R, et al. Functional and structural changes in the memory network associated with left temporal lobe epilepsy. Hum. Brain. Mapp 30(12), 4070-4081 (2009).

58. Voets NL, Zamboni G, Stokes MG, et al. Aberrant functional connectivity in dissociable hippocampal networks is associated with deficits in memory. J. Neurosci 34(14), 49204928 (2014).

59. Li H, Ji C, Zhu L, et al. Reorganization of anterior and posterior hippocampal networks associated with memory performance in mesial temporal lobe epilepsy. Clin. Neurophysiol 128(5), 830-838 (2017).

60. Fan X, Yan H, Shan Y, et al. Distinctive Structural and Effective Connectivity Changes of Semantic Cognition Network across Left and Right Mesial Temporal Lobe Epilepsy Patients. Neural. Plast 2016(1), 8583420 (2016).

61. Morgan VL, Sonmezturk HH, Gore JC, et al. Lateralization of temporal lobe epilepsy using resting functional magnetic resonance imaging connectivity of hippocampal networks. Epilepsia 53(9), 1628-1635 (2012).

62. Waites AB, Briellmann RS, Saling MM, et al. Functional connectivity networks are disrupted in left temporal lobe epilepsy. Ann. Neurol 59(2), 335-343 (2006).

63. Powell HW, Parker GJ, Alexander DC, et al. Abnormalities of language networks in temporal lobe epilepsy. Neuroimage 36(1), 209-221 (2007).

64. Thivard L, Hombrouck J, du Montcel ST, et al. Productive and perceptive language reorganization in temporal lobe epilepsy. Neuroimage 24(3), 841-851 (2005).

65. Vaessen MJ, Braakman HM, Heerink JS, et al. Abnormal modular organization of functional networks in cognitively impaired children with frontal lobe epilepsy. Cereb. Cortex 23(8), 1997-2006 (2013).

66. Wandschneider B, Centeno M, Vollmar C, et al. Risk-taking behavior in juvenile myoclonic epilepsy. Epilepsia 54(12), 2158-2165 (2013).

67. F Xiao, D An, D Lei, et al. Real-time effects of centrotemporal spikes on cognition in rolandic epilepsy, An EEG-fMRI study. Neurology 86(6), 544-551 (2016).

68. Kanner AM. Depression in epilepsy, a complex relation with unexpected consequences. Curr Opin Neurol 21(2), 190-194 (2008).

69. Fang P, An J, Zeng LL, et al. Mapping the convergent temporal epileptic network in left and right temporal lobe epilepsy. Neurosci. Lett 639(1), 179-184 (2017).

70. Chen S, Wu X, Lui S, et al. Resting-state fMRI study of treatment-naive temporal lobe epilepsy patients with depressive symptoms. Neuroimage 60(1), 299-304 (2012).

71. Kemmotsu N, Kucukboyaci NE, Leyden KM, et al. Frontolimbic brain networks predict depressive symptoms in temporal lobe epilepsy. Epilepsy. Res 108(9), 1554-1563 (2014).

72. Garcia-Ramos C, Lin JJ, Bonilha L, et al. Disruptions in cortico-subcortical covariance networks associated with anxiety in new-onset childhood epilepsy. Neuroimage. Clin 12(1), 815-824 (2016).

73. Braakman HM, van der Kruijs SJ, Vaessen MJ, et al. Microstructural and functional MRI studies of cognitive impairment in epilepsy. Epilepsia 53(10), 1690-1699 (2012). 